Pacific Journal of Mathematics

UNIQUENESS AND EXISTENCE PROPERTIES OF BOUNDED 


\title{
UNIQUENESS AND EXISTENCE PROPERTIES OF BOUNDED OBSERVABLES
}

\author{
STANLEY P. GUDDER
}

\begin{abstract}
Until recently observables have been nothing more than self-adjoint operators. However, due to axiomatic formulations of quantum mechanics, observables have now been placed in a more abstract setting. With the advent of this abstract concept comes the natural questions concerning uniqueness and existence. The uniqueness problem considered here seeks to answer the question: if two bounded observables have the same expectations in every state, are the observables equal? We say that an observable $z$ is the sum of two bounded observables $x$ and $y$ if the expectation of $z$ is the sum of the expectations of $x$ and $y$ for every state. The existence problem would pose the question: does the sum of two bounded observables exist? The author has found only partial answers to these questions. It is shown that the uniqueness property holds for simultaneous observables and certain classes of nonsimultaneous or complementary observables. The existence property holds for simultaneous observables, and a counterexample is given to show that this property does not hold in general. The last section of this paper considers systems in which the existence and uniqueness properties are known to hold.
\end{abstract}

Besides being mathematically interesting, the uniqueness and existence properties are also important from the physical standpoint. Due to the Bohr correspondence principle, the expectation value of an observable $x$ in a state $m$ is the value one would obtain by measuring $x$ using a classical experiment. Thus the collection of expectations of $x$ would be the set of classical values which an observable $x$ may attain. The uniqueness property would say that if two bounded observables are classically equivalent, then they are also equal in the quantum mechanical sense. The existence property would say that the sum of two bounded quantum mechanical observables always exists in the sense defined above. This existence property is so important, that it is postulated in some models for quantum mechanics. $([3 ; 4])$.

Let us consider two examples which illustrate our problem. Let $(\Omega, S)$ be a measurable space. The states of this system are the probability measures on $S$ and the observables are essentially the measurable functions or random variables on $S$. (Cf. [5]). The uniqueness property may be stated: if $f$ and $g$ are bounded measurable functions, does $\int f d \mu=\int g d \mu$ for every probability measure $\mu$ imply 
$f=g$ ? The answer, which is yes, may be seen as follows. Let $p \in \Omega$ and $\mu$ a probability measure concentrated at $p$. That is, for each $\Lambda \in S, \mu(\Lambda)=1$ if and only if $p \in \Lambda$. Now it is easily seen that $\int_{\Lambda} f d \mu=\int_{\Lambda} g d \mu$ for each $\Lambda \in S$. Therefore, $f=g$ almost everywhere with respect to $\mu$, and in particular $f(p)=g(p)$. The existence problem is even more trivial since $f+g$ is a bounded measurable function and $\int(f+g) d \mu=\int f d \mu+\int g d \mu$ for all probability measures $\mu$. For our next example let $H$ be a Hilbert space with inner product $\langle\cdot, \cdot\rangle$. The observables are self-adjoint operators and the pure states are defined by unit vectors in the standard way. (Cf. [2;5]). The uniqueness problem is: if $A$ and $B$ are bounded self-adjoint operators, does $\langle\phi, A \phi\rangle=\langle\phi, B \phi\rangle$ for all $\phi \in H,\|\phi\|=1$ imply $A=B$ ? It is well known that the answer is yes, although the proof is not quite as simple as in the previous example. The existence problem is again trivially satisfied since $A+B$ is a bounded self-adjoint operator and $\langle\phi,(A+B) \phi\rangle=\langle\phi, A \phi\rangle+\langle\phi, B \phi\rangle$ for all $\phi \in H$.

2. Definitions and notation. Let $L$ be a partially ordered set with first and last elements 0,1 respectively which is closed under a complementation $a \rightarrow a^{\prime}$ satisfying

(i) $\left(a^{\prime}\right)^{\prime}=a$;

(ii) $a \leqq b$ implies $b^{\prime} \leqq a^{\prime}$.

We denote the least upper bound and greatest lower bound of $a, b \in L$, if they exist, by $a \vee b$ and $a \wedge b$ respectively, and assume

(iii) $a \vee a^{\prime}=1$ for all $a \in L$.

We say that $a, b \in L$ are disjoint and write $a \perp b$ if $a \leqq b^{\prime}$. If $a \perp b$ we write $a+b$ for $a \vee b$, and if $a \leqq b$ we write $b-a$ for $b \wedge a^{\prime}$. We say that $a, b \in L$ split and write $a \leftrightarrow b$ if there exist mutually disjoint elements $a_{1}, b_{1}, c \in L$ such that $a=a_{1}+c$ and $b=b_{1}+c$. We call $L$ a logic if it also satisfies

(iv) $\sum a_{i} \in L$ for any disjoint sequence $\left(a_{i}\right) \subset L$;

(v) if $a, b, c \in L$ mutually split, then $a \leftrightarrow b \vee c$.

The elements of a logic $L$ are called propositions. A state is a nonnegative function $m$ on $L$ satisfying

(i) $m(1)=1$;

(ii) $m\left(\sum a_{i}\right)=\sum m\left(a_{i}\right)$.

A logic is full in case

(1) if $a \neq b$ there exists a state $m$ such that $m(a) \neq m(b)$;

(2) if $a \neq 0$ there exists a state $m$ such that $m(a)=1$.

We shall henceforth assume that $L$ has at least three (and hence four) distinct elements. A logic is quite full if the statement $m(b)=1$ whenever $m(a)=1$ implies the statement $a \leqq b$. One should note that the two examples considered in the introduction are specific cases of 
quite full logics.

Our first lemma shows that a quite full logic is not only full but that the states preserve order.

Lemma 2.1. Let $L$ be a quite full logic.

(i) There exist states on $L$.

(ii) If $a \neq 0$ there exists $m \in M$ such that $m(a)=1$.

(iii) $a=b$ if and only if $m(a)=m(b)$ for all $m \in M$.

(iv) $a \leqq b$ if and only if $m(a) \leqq m(b)$ for all $m \in M$.

Proof. (i) If there were no states on $L$ then the following statement would hold vacuously: $m(0)=1$ whenever $m(1)=1$. Hence $1 \leqq 0$ and $1=0$.

(ii) If $m(\alpha)<1$ for every $m \in M$, then the following statement would hold vacuously: $m(0)=1$ whenever $m(a)=1$. Hence $a=0$ and there are only two elements in $L$.

(iii) will follow from (iv). If $a \leqq b$ then $b=a+b \wedge a^{\prime}$ and $m(a)=m(b)-m\left(b \wedge a^{\prime}\right) \leqq m(b)$. If $m(a) \leqq m(b)$ for every $m \in M$, then $m(b)=1$ whenever $m(a)=1$ and $a \leqq b$.

An observable $x$ is a map from the Borel sets $B(R)$ of the real line $R$ into a full logic $L$ which satisfies

(1) $x(R)=1$;

(2) $x(E) \perp x(F)$ if $E \cap F=\varnothing$;

(3) $x\left(\cup E_{i}\right)=\sum x\left(E_{i}\right)$, if $E_{i} \cap E_{j}=\varnothing, i \neq j$.

A collection of observables $\left\{x_{\lambda}: \lambda \in \Lambda\right\}$ is simultaneous if $x_{\lambda}(E) \leftrightarrow x_{\mu}(F)$ for all $E, F \in B(R)$ and $\lambda, \mu \in \Lambda$. If $x$ is an observable and $u$ a Borel function on $R$, we define the observable $u(x)$ by $u(x)(E)=x\left[u^{-1}(E)\right]$ for all $E \in B(R)$. More generally, if $\psi$ is an $n$-dimensional Borel function and $u_{1}, \cdots, u_{n}$ are Borel functions on $R$, we define the observable $\psi\left(u_{1}(x), \cdots, u_{n}(x)\right)$ by

$$
\psi\left(u_{1}(x), \cdots, u_{n}(x)\right)(E)=x\left\{\omega: \psi\left(u_{1}(\omega), \cdots, u_{n}(\omega)\right) \in E\right\}
$$

for all $E \in B(R)$.

The spectrum $\sigma(x)$ of an observable $x$ is the smallest closed set $E$ such that $x(E)=1$. An observable is bounded is $\sigma(x)$ is bounded. The norm of a bounded observable $x$ if $|x|=\sup \{|\lambda|: \lambda \in \sigma(x)\}$. The expectation of an observable $x$ in the state $m$ is

$$
m(x)=\int \lambda m[x(d \lambda)]
$$

if the integral exists.

3. Observables with finite spectra. In this section we collect some elementary results which will be used in the sequel. Most of the proofs are routine and we leave their verification to the reader. 
It is easily seen that if an observable $x$ has countable spectrum $\sigma(x)=\left\{\lambda_{1}, \lambda_{2}, \cdots\right\}$, then $x$ has the form $x(E)=\sum\left\{x\left(\lambda_{i}\right): \lambda_{i} \in E\right\}$. The converse does not hold, however, as may be seen from the following result.

LEMMA 3.1. If there is a countable set $K=\left\{\lambda_{1}, \lambda_{2}, \cdots\right\} \subset R$ such that $x(E)=\sum\left\{x\left(\lambda_{i}\right): \lambda_{i} \in E\right\}$ for every $E \in B(R)$ and $x\left(\lambda_{i}\right) \neq 0, i=$ $1,2, \cdots$, then $\sigma(x)=\mathrm{Cl} K$.

As a consequence we have:

COROLlaRY 3.2. An observable $x$ has finite spectrum $\sigma(x) \subset\left\{\lambda_{1}\right.$, $\left.\cdots, \lambda_{n}\right\}$ if and only if $x(E)=\sum\left\{x\left(\lambda_{i}\right): \lambda_{i} \in E\right\}$ for all $E \in B(R)$.

It is easily seen that the range of an observable is a Boolean sub $\sigma$-algebra of $L$. For this reason, if an observable has infinite spectrum, its range cannot be countable even if its spectrum is. In the finite case we have:

THEOREM 3.3. An observable $x$ has a finite range if and only if $\sigma(x)$ is a finite set.

Proof. The sufficiency follows from Corollary 3.2. To prove necessity, let $\widetilde{R}=\left\{a_{1}, \cdots, a_{n}\right\}$ be the range of $x$. Let $K=\left\{c_{1}, \cdots, c_{m}\right\}$ be the set of distinct nonzero minimal elements of $\widetilde{R}$, i.e., $a \in K$ if and only if $0 \neq a \in \widetilde{R}$, and if $0 \neq c \in \widetilde{R}$ with $c \leqq a$, then $c=a$. Now $c_{i} \wedge c_{j}=0, i \neq j$, since if $c_{i} \wedge c_{j}=a \neq 0$, then $a \in \widetilde{R}$ and $a<c_{i}$, a contradiction. Since $\widetilde{R}$ is a Boolean $\sigma$-algebra, $c_{i} \perp c_{j}, i \neq j$. Since $\widetilde{R}$ is finite, every nonzero element of $\widetilde{R}$ is " $\geqq$ " at least one element of $K$. Now let $a \in \widetilde{R}$ and $D=\left\{d_{i}: d_{i} \in K, d_{i} \leqq a\right\}$. Then $a=\sum d_{i}$, since if not $0 \neq d=a-\sum d_{i} \in \widetilde{R}$, and there is a $c \in K$ with $c \leqq d \leqq a$, but $c \notin D$, a contradiction. We now claim that there are numbers $\lambda_{j} \in R$ such that $x\left(\lambda_{j}\right)=c_{j}, j=1, \cdots, m$. Suppose not, then there is a Borel set $E \in B(R)$ having more than one point such that $c_{j}=x(E)$. For a positive integer $n$ we have $c_{j}=x(E \cap[-n, n])+x\left(E \cap[-n, n]^{\prime}\right)$. One of the propositions on the right must be zero and the other $c_{j}$. As $n$ increases, the infinite term must eventually be zero since otherwise $c_{j}=\Lambda_{n} x\left(E \cap[-n, n]^{\prime}\right)=x\left(E \bigcap_{n}[-n, n]^{\prime}\right)=x(\phi)=0$. Hence $c_{j}=x(E \cap[-n, n])=x(E \cap[-n, 0])+x(E \cap(0, n])$ and we again have one of the terms equal to 0 . Suppose, for definiteness, the first term is zero. Then $c_{j}=x(E \cup[0, n])$. Continuing in this manner, by taking smaller closed intervals, and applying a theorem on closed nested intervals, there exists a number $\lambda_{j}$ such that $c_{j}=x\left(E \cap\left\{\lambda_{j}\right\}\right)=x\left(\lambda_{j}\right)$. We then have $x\left(\cup\left\{\lambda_{j}\right\}\right)=\sum x\left\{\lambda_{j}\right\}=\sum c_{j}=1$ and $\sigma(x)=\cup\left\{\lambda_{j}\right\}$.

CoRollary 3.4. If an observable $x$ has a finite range $\widetilde{R}$, there 
exists an integer $n$ such that $\widetilde{R}$ has $2^{n}$ elements and $\sigma(x)$ has $n$ elements.

An observable $x$ is a proposition observable if $\sigma(x) \subset\{0,1\}$. If $y$ is a proposition observable and $y(\{1\})=a$ we denote $y$ by $x_{a}$. If, in particular, $a=x(E)$ for some observable $x$, we denote $y$ by $x_{E}$. Of particular importance are the observables $I=x_{1}$ and $0=x_{0}$. Notice that if $I_{E}$ is the indication function of a set $E \in B(R)$, that $I_{E}(x)=x_{E}$.

LEMMA 3.5. An observable $x$ has spectrum $\sigma(x) \subset\left\{\lambda_{1}, \cdots, \lambda_{n}\right\}$ if and only if $x=\sum \lambda_{i} x_{\left\{\lambda_{i}\right\}}$.

LEMMA 3.6. The following statements are equivalent:

(i) $x$ is a proposition observable

(ii) $x$ is an indicator function of an observable;

(iii) $x^{2}=x$.

4. Uniqueness properties. The following is proved in [1].

TheOREM 4.1. $V(x)=\mathrm{Cl}\{m(x): m \in M\}$ is the smallest closed interval containing $\sigma(x)$.

In the sequel $x, y, z$ will denote bounded observables. We first prove a weak uniqueness property which holds for all $x, y$.

LEMMA 4.2. If $m[u(x)]=m[u(y)]$ for every Borel function $u$ and every $m$ in a full logic, then $x=y$.

Proof. If $E \in B(R)$, then for every $m \in M, m[x(E)]=m\left[I_{E}(x)\right]=$ $m\left[I_{E}(y)\right]=m[y(E)]$. Since $L$ is full, $x(E)=y(E)$ and $x=y$.

We now turn to the stronger uniqueness property: if $m(x)=m(y)$ then $x=y$.

LEMMA 4.3. If $x$ and $y$ are simultaneous on a full logic and $m(x)=m(y)$ for every $m \in M$, then $x=y$.

Proof. Since $x$ and $y$ are simultaneous $m(x-y)=0$. (Theorem 6.1. [1]). Therefore $V(x-y)=0$ and by Theorem $4.1 \sigma(x-y)=0$. Hence $x-y=0$ and $x=y$.

LEMMA 4.4. If $x$ and $y$ are observables on a full logic with one or two point spectra and $m(x)=m(y)$ for all $m \in M$, then $x=y$.

Proof. Since $V(x)=V(y)$ we have $\sigma(x)=\sigma(y)$. Hence if either $x$ or $y$ has one point spectrum $x=y$. Now suppose both $x$ and $y$ have two point spectrum $\left\{\lambda_{1}, \lambda_{2}\right\}$. By Lemma 3.5, $x=\lambda_{1} x_{\left\{\lambda_{1}\right\}}+\lambda_{2} x_{\left\{\lambda_{2}\right\}}$ 
and $y=\lambda_{1} y_{\left\{\lambda_{1}\right\}}+\lambda_{2} y_{\left\{\lambda_{2}\right\}}$. Hence for all

$$
m \in M, \lambda_{1} m\left[x\left(\lambda_{1}\right)\right]+\lambda_{2} m\left[x\left(\lambda_{2}\right)\right]=\lambda_{1} m\left[y\left(\lambda_{1}\right)\right]+\lambda_{2} m\left[y\left(\lambda_{2}\right)\right] .
$$

Since $x\left(\lambda_{1}\right)+x\left(\lambda_{2}\right)=y\left(\lambda_{1}\right)+y\left(\lambda_{2}\right)=1$ we have

$$
m\left[x\left(\lambda_{1}\right)\right]\left(\lambda_{1}-\lambda_{2}\right)=m\left[y\left(\lambda_{1}\right)\right]\left(\lambda_{1}-\lambda_{2}\right)
$$

and $m\left[x\left(\lambda_{1}\right)\right]=m\left[y\left(\lambda_{1}\right)\right]$. Hence $x\left(\lambda_{1}\right)=y\left(\lambda_{1}\right)$ and $x=y$.

A number $\lambda \in \sigma(x)$ is the largest (smallest) spectral point of $x$ if $\lambda \geqq \mu(\lambda \leqq \mu)$ for all $\mu \in \sigma(x)$. It is trivial that every bounded observable has a unique largest (smallest) spectral point.

THEOREM 4.5. Let $m(x)=m(y)$ for every $m \in M$ on a quite full logic. If $\lambda_{1}$ and $\mu_{1}$ are the largest spectral points of $x$ and $y$ respectively, then $\lambda_{1}=\mu_{1}$ and $x\left(\lambda_{1}\right)=y\left(\mu_{1}\right)$.

Proof. Since $V(x)=V(y)$ we have $\lambda_{1}=\mu_{1}$. Now we may assume that the spectra of $x$ and $y$ are positive, since if not we consider $x^{(1)}=x-\nu I$ and $y^{(1)}=y-\nu I$ where $\nu$ is the common smallest spectral point of $x$ and $y$. Then $\sigma\left(x^{(1)}\right)=\sigma(x)-\nu \geqq 0$ with a similar inequality for $y^{(1)}$ and $m\left(x^{(1)}\right)=m\left(y^{(1)}\right)$ for all $m \in M$. Now the largest spectral point of $x^{(1)}$ and $y^{(1)}$ is $\lambda_{1}-\nu$ and if the theorem is proved for $x^{(1)}$ and $y^{(1)}$ we have

$$
x\left(\lambda_{1}\right)=\left(x^{(1)}+\nu I\right)\left(\lambda_{1}\right)=x^{(1)}\left(\lambda_{1}-\nu\right)=y^{(1)}\left(\lambda_{1}-\nu\right)=y\left(\lambda_{1}\right) .
$$

Now suppose we have $m\left[x\left(\lambda_{1}\right)\right]=1$. Letting $\lambda_{1}>\mu_{2} \in \sigma(y)$ and $E=\sigma(y)-\left\{\lambda_{1}, \mu_{2}\right\}$ we obtain

$$
\lambda_{1}=m(x)=m(y)=\lambda_{1} m\left[y\left(\lambda_{1}\right)\right]+\mu_{2} m\left[y\left(\mu_{2}\right)\right]+\int_{E} \lambda m_{y}(d \lambda) .
$$

Since $m\left[y\left(\mu_{2}\right)\right]=1-m\left[y\left(\lambda_{1}\right)\right]-\int_{E} m_{y}(d \lambda)$, (1) may be written in the form

$$
m\left[y\left(\lambda_{1}\right)\right]=1+\left(\lambda_{1}-\mu_{2}\right)^{-1} \int_{E}\left(\mu_{2}-\lambda\right) m_{y}(d \lambda) .
$$

If $\lambda_{1}$ is an isolated point of $\sigma(y)$, we may take $\mu_{2} \geqq \mu$ for every $\mu \in \sigma(y)-\left\{\lambda_{1}\right\}$. The second term on the right of (2) is then nonnegative. Since $m\left[y\left(\lambda_{1}\right)\right] \leqq 1$, this term is zero and $m\left[y\left(\lambda_{1}\right)\right]=1$. Suppose, on the other hand, that $\lambda_{1}$ is not isolated and that $m\left[y\left(\lambda_{1}\right)\right] \neq 1$. Then there exists $\lambda_{1}>\mu^{0} \in \sigma(y)$ such that $N=\int_{(0, \mu)} m_{y}(d \lambda)>0$. Now let $\mu^{1} \in \sigma(y)$ satisfy $\mu^{0}<\mu^{1}<\lambda_{1}$ and let $\delta=\left(\mu^{1}-\mu^{0}\right) N>0$. We now find $\mu_{2} \in \sigma(y)$ which satisfies:

(i) $\mu^{1}<\mu_{2}<\lambda_{1}$; 
(ii) $\quad\left(\lambda_{1}-\mu_{2}\right)<\mu_{2}$;

(iii) $\int_{\left(\mu_{2}, \lambda_{1}\right)} \lambda m_{y}(d \lambda)<\delta$.

We then obtain

$$
\begin{aligned}
\int_{E}\left(\mu_{2}-\lambda\right) m_{y}(d \lambda) & =\left(\int_{(0, \mu 0)}+\int_{\left(\mu_{0}, \mu_{2}\right)}+\int_{\left(\mu_{2}, \lambda_{1}\right)}\right)\left(\mu_{2}-\lambda\right) m_{y}(d \lambda) \\
& \geqq\left(\int_{(0, \mu 0)}+\int_{\left(\mu_{2}, \lambda_{1}\right)}\right)\left(\mu_{2}-\lambda\right) m_{y}(d \lambda) \\
& \geqq\left(\mu_{2}-\mu^{0}\right) \int_{(0, \mu 0)} m_{y}(d \lambda)+\int_{\left(\mu_{2}, \lambda_{1}\right)}\left(\mu_{2}-\lambda\right) m_{y}(d \lambda) \\
& \geqq \delta-\int_{\left(\mu_{2}, \lambda_{1}\right)}\left(\lambda-\mu_{2}\right) m_{y}(d \lambda) \geqq \delta-\int_{\left(\mu_{2}, \lambda_{1}\right)} \lambda m_{y}(d \lambda)>0 .
\end{aligned}
$$

This gives a contradiction, and hence $m\left[y\left(\lambda_{1}\right)\right]=1$. Since $L$ is quite full $x\left(\lambda_{1}\right) \leqq y\left(\lambda_{1}\right)$. By symmetry $x\left(\lambda_{1}\right)=y\left(\lambda_{1}\right)$.

CoRollary. If $m(x)=m(y)$ for all $m \in M$ on a quite full logic, then $x(\lambda)=y(\lambda)$ for all $\lambda \in R$.

Proof. Let $x^{(1)}=x-\left(\lambda_{1}-\lambda\right) I$ and $y^{(1)}=y-\left(\lambda_{1}-\lambda\right) I$. Then $m\left(y^{(1)}\right)=m\left(y^{(1)}\right)$ for all $m \in M$ and the largest spectral point of $x^{(1)}$ and $y^{(1)}$ is $\lambda$.

THEOREM 4.6. If $x$ has countable spectrum and $m(x)=m(y)$ for all $m \in M$ on a quite full logic, then $x=y$.

Proof. Let $\sigma(x)=\left\{\lambda_{i}: i=1,2, \cdots\right\}$. Applying the previous corollary, $y(\sigma(x))=\sum y\left(\lambda_{i}\right)=\sum x\left(\lambda_{i}\right)=1$. Therefore $\sigma(y) \subset \sigma(x)$ and $\sigma(y)$ is countable. Therefore $y$ has the form

$$
y(E)=\sum\left\{y\left(\mu_{i}\right): \mu_{i} \in \sigma(y) \cap E\right\}=\sum\left\{x\left(\lambda_{i}\right): \lambda_{i} \in \sigma(x) \cap E\right\}=x(E) .
$$

Notice that the examples in the introduction show that Theorem 4.6 holds for arbitrary bounded observables in case $L$ is the lattice of closed subspaces of a Hilbert space or a $\sigma$-field of subsets of a set.

5. Existence properties. We recall that $z$ is the sum of $x$ and $y$ if $m(z)=m(x)+m(y)$ for every $m \in M$. In this case we write $z=x+y$.

Lemma 5.1. If $x$ and $y$ are simultaneous then $x+y$ exists.

Proof. This follows from Theorem 3.3 [5].

Notice again that the examples in the introduction show that the 
sum of two arbitrary bounded observables always exists in case $L$ is the lattice of closed subspaces of a Hilbert space or a $\sigma$-field of subsets of a set.

The rest of this section is directed toward finding an example of two complementary observables whose sum does not exist.

An anti-lattice is a complemented lattice in which the supremum of any two nonzero elements is 1 . It is easily seen that an anti-lattice is a quite full logic.

Lemma 5.2. A Boolean subalgebra of an anti-lattice $L$ can have at most 4 elements.

Proof. Let $a, b$ be distinct elements of $L$ not equal to 0 or 1 and $a \neq b^{\prime}$. We now show that $a$ and $b$ do not split. Suppose, on the contrary, that $a=a_{1}+c$ and $b=b_{1}+c$ where $a_{1} \perp b_{1}$. Now neither $a_{1}$ nor $b_{1}$ is 0 , hence $c=0$ since otherwise $a_{1}+c=1$. Therefore $a \perp b$. But this is impossible since then $b^{\prime}=a \vee b^{\prime}=1$. The result follows since the elements of a Boolean algebra must split.

CoROLlary. Every observable on an anti-lattice is of the form $x=\lambda_{1} x_{a}+\lambda_{2} x_{a^{\prime}}$, where $\sigma(x)=\left\{\lambda_{1}, \lambda_{2}\right\}$.

Proof. This follows from Corollary 3.4, Lemma 3.5, and Lemma 5.2 .

We may also conclude the uniqueness property from Lemma 5.2.

CoROllaRY. If $m(x)=m(y)$ for all $m \in M$ on an anti-lattice, then $x=y$.

THEOREM 5.3. If $x$ and $y$ are complementary observables on an anti-lattice, then $x+y$ does not exist.

Proof. Suppose $a$ and $b$ do not split and that $z=x_{a}+x_{b}$. By the first corollary of Lemma $5.2, z=\lambda_{1} x_{c}+\lambda_{2} x_{c^{\prime}}$. Therefore

$$
\left(\lambda_{1}-\lambda_{2}\right) m(c)+\lambda_{2}=m(a)+m(b)
$$

for every $m \in M$. Now it is easily seen that on an anti-lattice $L$, given $0<\alpha<1$ and $a \in L$, there exists a state $m$ such that $m(\alpha)=\alpha$ and $m(b)=0$ for $b \neq a, a^{\prime}, 1$. Suppose $c=a$. Letting $m$ be a state which is 0 on $b$ and 1 on $a$ we have $\lambda_{1}=1$. Letting $m$ be a state which is 0 on $a$ and 1 on $b$ we have $\lambda_{2}=1$ and hence $m(a)+m(b)=1$ for all $m \in M$ which is impossible. Hence $c \neq a$, and in a similar way $c \neq a^{\prime}, b, b^{\prime}, 0,1$. Now let $m$ satisfy $m(\alpha)=\alpha \neq \lambda_{2}$ and $m(c)=$ 
$m(b)=0$. This gives a contradiction and hence $z$ does not exist. Now suppose $x+y$ exists. It must be of the form

$$
\begin{aligned}
x+y & =\lambda_{1} x_{a}+\lambda_{2} x_{a^{\prime}}+\mu_{1} x_{b}+\mu_{2} x_{b^{\prime}} \\
& =\left(\lambda_{1}-\lambda_{2}\right) x_{a}+\left(\mu_{1}-\mu_{2}\right) x_{b}+\left(\lambda_{2}+\mu_{2}\right) I
\end{aligned}
$$

where $a \leftrightarrow b$. Since $\left(\lambda_{2}+\mu_{2}\right) I$ is simultaneous with every observable, $x+y-\left(\lambda_{2}+\mu_{2}\right) I$ exists. But this contradicts the previous work.

We next give an example of a quite full logic in which the sum of certain complementary observables exist while the sum of others do not. Let $L_{1}$ and $L_{2}$ be logics. The direct product $L_{1} \otimes L_{2}$ is the set $\left\{\left(a_{1}, a_{2}\right): a_{1} \in L_{1}, a_{2} \in L_{2}\right\}$ with the partial ordering $\left(a_{1}, b_{1}\right) \leqq\left(a_{2}, b_{2}\right)$ if $a_{1} \leqq a_{2}$ and $b_{1} \leqq b_{2}$. It is easy to check that $L_{1} \otimes L_{2}$ is a logic.

LEMMA 5.4. If $L_{1}$ and $L_{2}$ are logics with states $M_{1}, M_{2}$ respectively, then $m$ is a state on $L_{1} \otimes L_{2}$ if and only if there exist states $m_{1} \in M_{1}$, $m_{2} \in M_{2}$ and $0 \leqq \alpha \leqq 1$ such that $m(\alpha, b)=\alpha m_{1}(\alpha)+(1-\alpha) m_{2}(b)$.

Proof. The sufficiency is a routing check. To prove necessity, we first notice that $1=m(1,1)=m[(1,0)+(0,1)]=m(1,0)+m(0,1)$. Let us assume that $m(1,0), m(0,1) \neq 0$. Now define $m_{1}, m_{2}$ as $m_{1}(\cdot)=m(\cdot, 0) / m(1,0)$ and $m_{2}(\cdot)=m(0, \cdot) / m(0,1)$ respectively. Then $m_{1} \in M_{1}, m_{2} \in M_{2}$ and

$$
\begin{aligned}
m(a, b) & =m[(a, 0)+(0, b)]=m(a, 0)+m(0, b) \\
& =(1,0) m_{1}(a)+[1-m(1,0)] m_{2}(b) .
\end{aligned}
$$

Since $m(1,0)$ and $m(0,1)$ cannot both be zero, suppose, for definiteness, that $m(0,1)=0$. Then since $(0, a) \leqq(0,1)$ we have $m(0, a)=0$ for all $a \in L_{2}$. Now define $m_{1}(\cdot)=m(\cdot, 0)$. Then $m_{1} \in M_{1}$ and we have $m(a, b)=m(a, 0)=m_{1}(a)$.

CoRollaRY. If $L_{1}$ and $L_{2}$ are full (quite full) logics, so is $L_{1} \otimes L_{2}$.

Lemma 5.5. A necessary and sufficient condition for $z$ to be an observable on $L_{1} \otimes L_{2}$ is that there exist observables $x, y$ on $L_{1}, L_{2}$ respectively such that $z(E)=(x(E), y(E))$.

Proof. The sufficiency is routine. For necessity, let $z$ be an observable on $L_{1} \otimes L_{2}$ and define $x(\cdot), y(\cdot)$ as $(x(E), y(E))=z(E)$. It is easy to check that $x$ and $y$ are observables on $L_{1}$ and $L_{2}$ respectively.

We now construct our example. Let $L_{1}$ be the quite full logic discussed in the second example in the introduction and let $L_{2}$ be an anti-lattice. Denote observables on $L_{1}$ and $L_{2}$ by $x^{(1)}, y^{(1)}, \cdots$, and 
$x^{(2)}, y^{(2)}, \ldots$ respectively. It is easily seen that the sum of two complementary observables of the form $\left(x^{(1)}, I^{(2)}\right)$ and $\left(y^{(1)}, I^{(2)}\right)$ exists, while the sum of two complementary observables of the form $\left(I^{(1)}, x^{(2)}\right)$ and $\left(I^{(1)}, y^{(2)}\right)$ does not.

One further remark seems to be in order. If it turns out that the uniqueness property does not hold, then even if the sum of two bounded observables exists it may not be unique. We can show this by an example. Suppose $x$ and $y$ are distinct bounded observables and that $m(x)=m(y)$ for all $m \in M$. Now $2 x=x+y$ and $2 y=x+y$ is easy to check, and hence $x+y$ exists but is not unique.

6. Logics with uniqueness and existence properties. Let $L$ be an arbitrary logic, (i.e. $L$ is not necessarily full.) If the bounded observables on $L$ have the uniqueness property, then $L$ is said to have Property $U$. If the bounded observables on $L$ have the existence property, then $L$ is said to have Property $E$. In the previous paragraph we noted that if $L$ has Property $E$ but not $U$ then sums need not be unique. If however, $L$ has Property $E$ and the sums are unique, then $L$ has Property $U$. Indeed, suppose $L$ possesses unique sums and $m(x)=m(y)$ for every state. Then $x$ and $y$ are both the sum of $y$ and 0 and hence $x=y$. Now it is not unreasonable to assume that quantum mechanical logics possess unique sums and hence both properties $U$ and $E$. Mackey has speculated Property $U$ by his Axiom II' [2] and this property is a consequence of Segal's axiomatic formulation of quantum mechanics [4]. Property $E$ is postulated in Segal's model [4] and von Neumann's model [3]. In this section we shall give some consequences of Properties $U$ and $E$ as far as the algebraic and analytic structure of our present system is concerned. This is only a preliminary study of this subject and a deeper study may prove fruitful. We shall close this section with some questions which may lead to interesting results in this direction.

The proof of the following lemma is trivial.

Lemma 6.1. If $L$ has Property $U$, then (iii) of Lemma 2.1 holds.

It is not known whether Property $U$ implies (ii) and (iv) of Lemma 2.1 or more specifically whether $L$ is full or quite full.

Lemma 6.2. If $L$ is quite full and has Property $E$, then $L$ is a lattice. In fact $a \wedge b=\left(x_{a}+x_{b}\right)(\{2\})$.

Proof. Let $z=x_{a}+x_{b}$. Notice that $0 \leqq m(z) \leqq 2$ for every state $m$ and hence by Theorem 3.1, $\sigma(z) \subset[0,2]$. Let $c=z(\{2\})$ and suppose $m(c)=1$. Then $m(z)=2$ and $m(a)=m(b)=1$. Hence $c \leqq a, c \leqq b$. 
Now suppose $d \leqq a, d \leqq b$. Then $m(d)=1$ implies $m(z)=2$ which implies $m(c)=1$. Thus $d \leqq c$ and $c=a \wedge b$.

Corollary 6.3. If $L$ is quite full and has Property $E$, then

(i) $\left[\left(x_{a}+x_{b}\right)(\{0\})\right]^{\prime}=a \wedge b$.

(ii) $\left(x_{a}-x_{b}\right)(\{1\})=a \wedge b^{\prime}$.

(iii) if $a, b, \cdots, c$ are a finite number of elements of $L$,

$$
\left(x_{a}+x_{b}+\cdots+x_{c}\right)(\{2\})=a \wedge b \wedge \cdots \wedge c .
$$

Corollary 6.4. Suppose $L$ is quite full and has Property E. If $m(a)=m(b)=1$, then $m(a \wedge b)=1$. If $m(a)=m(b)=0$, then $m(a \vee b)=0$.

It is shown in [6] that if a full logic $L$ is a lattice and satisfies the conclusion of Corollary 6.4, then $L$ is quite full. In the remainder of this section we shall assume that $L$ is quite full and has Properties $U$ and $E$. It follows that the set of bounded observables $X$ of $L$ is a linear space over the field of real numbers.

THEOREM 6.5. (i) $X$ is a normed linear space.

(ii) Denoting the closed convex hull of a set $E$ by $\mathrm{CH}(E)$,

$$
\mathrm{CH}[\sigma(x+y)] \subset \mathrm{CH}[\sigma(x)]+\mathrm{CH}[\sigma(y)] \text {. }
$$

Proof. (i) Applying Theorem 6.1 [1],

$$
\begin{aligned}
|x+y| & =\sup \{|m(x+y)|: \text { all } m\}=\sup \{|m(x)+m(y)|: \text { all } m\} \\
& \leqq \sup \{|m(x)|: \text { all } m\}+\sup \{|m(y)|: \text { all } m\}=|x|+|y| .
\end{aligned}
$$

(ii) Applying Theorem 3.1,

$$
\begin{aligned}
\mathrm{CH}[\sigma(x+y)] & =\{m(x+y): \text { all } m\} \subset\{m(x): \text { all } m\}+\{m(y): \text { all } m\} \\
& =\mathrm{CH}[\sigma(x)]+\mathrm{CH}[\sigma(y)] .
\end{aligned}
$$

The next theorems illustrate the intimate relation between propositions and proposition observables.

LEMMA 6.6. If $a \leftrightarrow b$, then $x_{a \wedge b}=x_{a} x_{b}$ and $x_{a \vee b}=x_{a}+x_{b}-x_{a} x_{b}$.

Proof. Applying Proposition 3.5 [5], there exists an observable $x$ and Borel sets $E, F$ such that $a=x(E), b=x(F)$. Therefore, $x_{a} x_{b}=I_{E}(x) I_{F}(x)=\left(I_{E} \cdot I_{F}\right)(x)=I_{E \cap F}(x)=x_{a \wedge b}$. The proof of the second statement is similar.

THEOREM 6.7. The following three statements are equivalent: 
(i) $a \perp b$.

(ii) $x_{a}+x_{b}$ is a proposition observable.

(iii) $x_{a}+x_{b}=x_{a \vee b}$.

Proof. If $a \perp b$, then applying the previous lemma $\left(x_{a}+x_{b}\right) \cdot\left(x_{a}+x_{b}\right)=x_{a}+x_{b}+2 x_{a} x_{b}=x_{a}+x_{b}+2 x_{a \wedge b}=x_{a}+x_{b}$.

It follows from Lemma 3.6 that $x_{a}+x_{b}$ is a proposition observable. Conversely, suppose $x_{a}+x_{b}$ is a proposition observable and that $m(a)=1$. Then $m\left(x_{a}+x_{b}\right)=1+m(b)$ and hence $m(b)=0$. Thus $a \leqq b^{\prime}$ and $a \perp b$. Therefore (i) and (ii) are equivalent. Certainly (iii) implies (ii). Finally (i) implies (iii) by the previous lemma.

The next theorem may be proved in a similar manner.

COROLLARY 6.8. The following three statements are equivalent:

(i) $a \leqq b$.

(ii) $x_{b}-x_{a}$ is a proposition observable.

(iii) $x_{b}-x_{a}=x_{b \wedge a^{\prime}}$.

Following von Neumann and Segal [3;4], we define a "multiplication" of two observables $x, y$ as: $x \circ y=1 / 2\left[(x+y)^{2}-x^{2}-y^{2}\right]$. Note that this multiplication reduces to the usual multiplication when $x$ and $y$ are simultaneous. This multiplication is commutative but, in general, it is not associative. In fact, let $L$ be the lattice of closed subspaces of a Hilbert space. Identifying the bounded observables $x, y, z$ with self-adjoint operators it is easily shown that $(x \circ y) \circ z=$ $x \circ(y \circ z)$ if and only if $y$ commutes with the commutator of $x$ and $z$ [3]. Two bounded observables $x, y$ are said to be compatible if $x \circ(z \circ y)=(x \circ z) \circ y$ for every $z \in X$.

Even the distributive law $(x+y) \circ z=x \circ z+y \circ z$ does not hold in general and there seems to be no physical reason for postulating it, although von Neumann does so. The distributive law is equivalent to the following: $(x+y)^{2}+(x-y)^{2}=2 x^{2}+2 y^{2}$ for all $x, y \in X$. If the distributive law were to hold, we could write our multiplication in the following equivalent forms:

$$
x \circ y=\frac{1}{2}\left[x^{2}+y^{2}-(x-y)^{2}\right]=\frac{1}{4}\left[(x+y)^{2}-(x-y)^{2}\right] .
$$

We close this section with a number of questions, for which it would be quite interesting to have answers. In Lemma 6.6 we showed that $a \leftrightarrow b$ implies $x_{a} \circ x_{b}=x_{a \wedge b}$.

(1) Does $x_{a} \circ x_{b}=x_{a \wedge b}$ imply $a \leftrightarrow b$ ?

(2) If $x_{a} \circ x_{b}$ is a proposition observable is $x_{a} \circ x_{b}=x_{a \wedge b}$ ? 
Note the similarity to Theorems $6.7,6.8$. The concept of compatibility was introduced by von Neumann and others.

(3) What is the physical significance of compatibility in our present system?

(4) What is the relationship, if any, between compatibility and simultaneity?

REMARK. After the final draft of this paper was completed, the author received a copy of the Argonne National Laboratory report 7065 by MD. MacLaren. In this report MacLaren assumes properties $U$ and $E$ and proves Lemma 6.2 under a slightly different axiomatic system.

\section{REFERENCES}

1. S. P. Gudder, Spectral methods for a generalized probability theory, Trans. Amer. Math. Soc. 119 (1965), 428-442.

2. G. W. Mackey, The Mathematical Foundations of Quantum Mechanics, W. A. Benjamin Inc., New York, 1963.

3. J. von Neumann, On an algebraic generalization of the quantum mechanical formalism (Part I), Mat. Sbornik 43 (1936), 515-584.

4. I. E. Segal, Postulates for general quantum mechanics, Ann. of Math. 48 (1947), 930-948.

5. V.S. Varadarajan, Probability in physics and a theorem on simultaneous observability, Comm. Pure Appl. Math. 15 (1962), 189-217.

6. N. Zierler, Axioms for non-relativistic quantum mechanics, Pacific J. Math. 12 (1961), 1151-1169.

Received July 27, 1965, and in revised form January 5, 1966. 



\section{PACIFIC JOURNAL OF MATHEMATICS}

\section{EDITORS}

\author{
H. SAMELSON \\ Stanford University \\ Stanford, California \\ J. P. JANS \\ University of Washington \\ Seattle, Washington 98105
}

\author{
J. DUGUNDJI \\ University of Southern California \\ Los Angeles, California 90007 \\ RICHARD ARENS \\ University of California \\ Los Angeles, California 90024
}

\section{ASSOCIATE EDITORS}
E. F. BECKENBACH
B. H. NeumanN
F. WOLF
K. YOSIDA

\section{SUPPORTING INSTITUTIONS}

\author{
UNIVERSITY OF BRITISH COLUMBIA \\ CALIFORNIA INSTITUTE OF TECHNOLOGY \\ UNIVERSITY OF CALIFORNIA \\ MONTANA STATE UNIVERSITY \\ UNIVERSITY OF NEVADA \\ NEW MEXICO STATE UNIVERSITY \\ OREGON STATE UNIVERSITY \\ UNIVERSITY OF OREGON \\ OSAKA UNIVERSITY \\ UNIVERSITY OF SOUTHERN CALIFORNIA
}

STANFORD UNIVERSITY

UNIVERSITY OF TOKYO

UNIVERSITY OF UTAH

WASHINGTON STATE UNIVERSITY

UNIVERSITY OF WASHINGTON

AMERICAN MATHEMATICAL SOCIETY CHEVRON RESEARCH CORPORATION TRW SYSTEMS

NAVAL ORDNANCE TEST STATION 


\section{Pacific Journal of Mathematics}

\section{Vol. 19, No. 1 \\ May, 1966}

A. R. Brodsky, The existence of wave operators for nonlinear equations... 1

Gulbank D. Chakerian, Sets of constant width................... 13

Robert Ray Colby, On indecomposable modules over rings with minimum condition....................................... 23

James Robert Dorroh, Contraction semi-groups in a function space ....... 35

Victor A. Dulock and Harold V. McIntosh, On the degeneracy of the Kepler

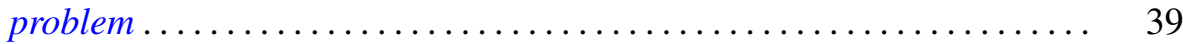

James Arthur Dyer, The inversion of a class of linear operators ......... 57

N. S. Gopalakrishnan and Ramaiyengar Sridharan, Homological dimension of Ore-extensions ................................. 67

Daniel E. Gorenstein, On a theorem of Philip Hall ................. 77

Stanley P. Gudder, Uniqueness and existence properties of bounded observables..................................... 81

Ronald Joseph Miech, An asymptotic property of the Euler function ....... 95

Peter Alexander Rejto, On the essential spectrum of the hydrogen energy and related operators ............................... 109

Duane Sather, Maximum and monotonicity properties of initial boundary

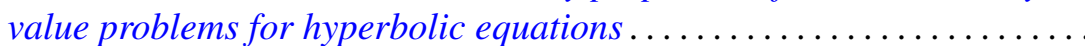

Peggy Strait, Sample function regularity for Gaussian processes with the parameter in a Hilbert space ........................... 159

Donald Reginald Traylor, Metrizability in normal Moore spaces ........... 175

Uppuluri V. Ramamohana Rao, On a stronger version of Wallis' formula ...............................

Adil Mohamed Yaqub, Some classes of ring-logics....... 